\title{
Iterative Schemes for Solving Mixed Variational-Like Inequalities ${ }^{1,2}$
}

\author{
Q. H. ANSARI ${ }^{3}$ AND J. C. YAO \\ Communicated by S. Schaible
}

\begin{abstract}
In the present paper, we introduce the concept of $\eta$-cocoercivity of a map and develop some iterative schemes for finding the approximate solutions of mixed variational-like inequalities. We use the concept of $\eta$-cocoercivity to prove the convergence of the approximate solutions to the exact solution of mixed variational-like inequalities.
\end{abstract}

Key Words. Mixed variational-like inequality problems, iterative schemes, $\eta$-cocoercive maps, $\eta$-strongly convex functions.

\section{Introduction}

Let $H$ be a real Hilbert space whose inner product and norm are denoted by $\langle\cdot, \cdot\rangle$ and $\|\cdot\|$, respectively. Let $K$ be a nonempty convex subset of $H$. Let $T, A: K \rightarrow H$ and $\eta: K \times K \rightarrow H$ be maps, and let $f: K \rightarrow \mathbb{R}$ be a realvalued function. We consider the mixed variational-like inequality problem (in short, MVLIP), which is to find $x^{*} \in K$ such that

$$
\left\langle T\left(x^{*}\right)-A\left(x^{*}\right), \eta\left(y, x^{*}\right)\right\rangle+f(y)-f\left(x^{*}\right) \geq 0, \quad \text { for all } y \in K .
$$

A random version of this problem is considered by Ding (Ref. 1). When $f(x)=0$ for all $x \in K$, problem (1) is studied by Noor (Ref. 2). When $A \equiv 0$, problem (1) reduces to the following problem considered by Dien (Ref. 3) and Noor (Ref. 4): Find $x^{*} \in K$ such that

$$
\left\langle T\left(x^{*}\right), \eta\left(y, x^{*}\right)\right\rangle+f(y)-f\left(x^{*}\right) \geq 0, \quad \text { for all } y \in K .
$$

\footnotetext{
${ }^{1}$ This research was supported by the National Science Council of the Republic of China.

${ }^{2}$ The authors express their sincere thanks to the anonymous referees for comments improving Lemma 2.1, which led to the present version of this paper.

${ }^{3}$ Reader, Department of Mathematics, Aligarh Muslim University, Aligarh, India.

${ }^{4}$ Professor, Department of Applied Mathematics, National Sun Yat-Sen University, Kaohsiung, Taiwan, ROC.
} 
When $f(x)=0$ for all $x \in K$ and $A \equiv 0$, problem (1) becomes the variationallike inequality problem studied in Refs. 5-9 with further applications. We remark that optimization problems involving $\eta$-convex functions can be formulated in the form of (MVLIP); see for example Refs. 3, 5, 7, 9.

To the best of our knowledge, most of the work on variational-like inequalities has been done only in the direction of the existence of solutions and their equivalence with optimization problems, mathematical programming problems, and other problems in operations research; see for example Refs. 1-9. Because of the applications of variational-like inequalities, it is necessary to develop techniques to find their solutions. In this direction, very few papers have appeared in the literature; see e.g. Refs. 2 and 4. This paper is an effort in this direction.

For classical optimization problems, Cohen (Refs. 10-11) and Cohen and Zhu (Ref. 12) introduced the so-called auxiliary problem principle as a general framework to describe and analyze computational algorithms ranging from gradient or subgradient algorithms to decomposition/coordination algorithms. Cohen (Ref. 13) further extended this approach to the computation of solutions to variational inequalities; the Cohen approach is close to the approach found in Ref. 14. Recently, Zhu and Marcotte (Ref. 15) analyzed the convergence behavior of iterative schemes based upon the auxiliary problem framework developed by Cohen (Ref. 13).

In the present paper, we introduce the concepts of $\eta$-cocoercivity, $\eta$ strong monotonicity, and $\eta$-strong convexity of a map, which generalize the definitions of cocoercivity (Ref. 16), strong monotonicity (Ref. 17), and strong convexity (Ref. 17), respectively. We see that $\eta$-cocoercivity is an intermediate concept that lies between $\eta$-strong monotonicity and $\eta$-monotonicity. Following the approach of Cohen (Ref. 13) and that of Zhu and Marcotte (Ref. 15), we develop some iterative schemes for finding the approximate solutions of the (MVLIP) and prove that these approximate solutions converge to the exact solution of the (MVLIP).

\section{Preliminaries}

Let $K$ be a nonempty subset of $H$, and let $F: K \rightarrow H$ and $\eta: K \times K \rightarrow H$ be two maps. Then $F$ is called:

(i) $\eta$-cocoercive, if there exists a constant $\alpha>0$ such that

$$
\langle F(x)-F(y), \eta(x, y)\rangle \geq \alpha\|F(x)-F(y)\|^{2}, \quad \text { for all } x, y \in K ;
$$

(ii) $\eta$-monotone, if

$$
\langle F(x)-F(y), \eta(x, y)\rangle \geq 0, \quad \text { for all } x, y \in K ;
$$


(iii) $\eta$-strongly monotone, if there exists a constant $\beta>0$ such that $\langle F(x)-F(y), \eta(x, y)\rangle \geq \beta\|x-y\|^{2}, \quad$ for all $x, y \in K ;$

(iv) $\eta$-relaxed monotone, if there exists a constant $\xi \geq 0$ such that $\langle F(y)-F(x), \eta(x, y)\rangle \geq \xi\|x-y\|^{2}, \quad$ for all $x, y \in K ;$

(v) Lipschitz continuous, if there exists a constant $\gamma>0$ such that

$$
\|F(x)-F(y)\| \leq \gamma\|x-y\|, \quad \text { for all } x, y \in K .
$$

When $\eta(x, y)=x-y$ for all $x, y \in K$, then the definitions (i), (ii), (iii), (iv) reduce to the definitions of cocoercivity (Ref. 16), monotonicity, strong monotonicity (Ref. 17), and relaxed monotonicity, respectively.

A map $\eta: K \times K \rightarrow H$ is called Lipschitz continuous, if there exists a constant $\lambda>0$ such that

$$
\|\eta(x, y)\| \leq \lambda\|x-y\|, \quad \text { for all } x, y \in K .
$$

It is clear that every $\eta$-cocoercive map is $\eta$-monotone, but that the converse is not true in general. Also, simple examples show that every $\eta$ cocoercive map need not be $\eta$-strongly monotone.

We note that every $\eta$-cocoercive map is also Lipschitz continuous provided that $\eta$ is Lipschitz continuous. Indeed,

$$
\begin{aligned}
\alpha\|F(x)-F(y)\|^{2} & \leq\langle F(x)-F(y), \eta(x, y)\rangle \\
& \leq\|F(x)-F(y)\| \cdot\|\eta(x, y)\| \\
& \leq \lambda\|F(x)-F(y)\| \cdot\|x-y\| .
\end{aligned}
$$

Hence,

$$
\|F(x)-F(y)\| \leq(\lambda / \alpha)\|x-y\|,
$$

and thus $F$ is Lipschitz continuous with constant $\lambda / \alpha$.

Every $\eta$-strongly monotone and Lipschitz continuous map is $\eta$-cocoercive, and it follows that $\eta$-cocoercivity is an intermediate concept that lies between $\eta$-monotonicity and $\eta$-strong monotonicity. In general, every $\eta$-monotone and Lipschitz continuous map need not be $\eta$-cocoercive.

A differentiable function $h: K \rightarrow \mathbb{R}$ on a convex set $K$ is called:

(i) $\eta$-convex (Ref. 18) if

$$
h(y)-h(x) \geq\left\langle h^{\prime}(x), \eta(y, x)\right\rangle, \quad \text { for all } x, y \in K,
$$

where $h^{\prime}(x)$ is the Fréchet derivative of $h$ at $x$;

(ii) $\eta$-strongly convex if there exists a constant $\mu>0$ such that

$$
h(y)-h(x)-\left\langle h^{\prime}(x), \eta(y, x)\right\rangle \geq(\mu / 2)\|x-y\|^{2}, \quad \text { for all } x, y \in K \text {. }
$$


It is easy to prove the following result.

Proposition 2.1. Let $h$ be a differentiable $\eta$-strongly convex functional on a convex subset $K$ of $H$, and let $\eta: K \times K \rightarrow H$ be a map such that $\eta(x, y)+\eta(y, x)=0$, for all $x, y \in K$. Then, $h^{\prime}$ is $\eta$-strongly monotone.

A map $F: K \rightarrow \mathbb{R}$ is called sequentially continuous at $x_{0}$ (Ref. 19), if $F\left(x_{k}\right) \rightarrow F\left(x_{0}\right)$ for all sequences $x_{k} \rightarrow x_{0} . F$ is called sequentially continuous on $K$, if it is sequentially continuous at each of its points.

Lemma 2.1. Let $\eta: K \times K \rightarrow H$ and $h^{\prime}$ be sequentially continuous from the weak topology to the weak topology and from the weak topology to the strong topology, respectively. Then, the map $g: K \rightarrow \mathbb{R}$, defined as $g(x)=$ $\left\langle h^{\prime}(x), \eta(y, x)\right\rangle$ for each fixed $y \in K$, is also sequentially continuous from the weak topology to the strong topology.

Proof. Let $\left\{x_{k}\right\}$ be a convergent (in the weak topology) sequence to $x$, which will be denoted by $x_{k} \rightarrow x$. Then,

$$
\left\|h^{\prime}\left(x_{k}\right)-h^{\prime}(x)\right\| \rightarrow 0 \quad \text { and } \quad \eta\left(y, x_{k}\right) \rightarrow \eta(y, x) .
$$

Now,

$$
\begin{aligned}
\left|g\left(x_{k}\right)-g(x)\right| & =\left|\left\langle h^{\prime}\left(x_{k}\right), \eta\left(y, x_{k}\right)\right\rangle-\left\langle h^{\prime}(x), \eta(y, x)\right\rangle\right| \\
& =\left|\left\langle h^{\prime}\left(x_{k}\right)-h^{\prime}(x), \eta\left(y, x_{k}\right)\right\rangle+\left\langle h^{\prime}(x), \eta\left(y, x_{k}\right)-\eta(y, x)\right\rangle\right| \\
& \leq\left\|h^{\prime}\left(x_{k}\right)-h^{\prime}(x)\right\| \cdot\left\|\eta\left(y, x_{k}\right)\right\|+\left|\left\langle h^{\prime}(x), \eta\left(y, x_{k}\right)-\eta(y, x)\right\rangle\right| .
\end{aligned}
$$

Since each weakly convergent sequence is bounded, we have

$$
\left|g\left(x_{k}\right)-g(x)\right| \rightarrow 0, \quad \text { as } k \rightarrow \infty .
$$

Hence, $g(x)$ is sequentially continuous from the weak topology to the strong topology.

For all $B \subseteq H$, we denote by $\operatorname{conv}(B)$ the convex hull of $B$. A point-toset map $G: H \rightarrow 2^{H}$ is called a KKM map if, for every finite subset $\left\{x_{1}, x_{2}, \ldots, x_{k}\right\}$ of $H$,

$$
\operatorname{conv}\left(\left\{x_{1}, x_{2}, \ldots, x_{k}\right\}\right) \subseteq \bigcup_{I=1}^{k} G\left(x_{i}\right) .
$$

In the next section, we shall use the following result.

Lemma 2.2. See Ref. 20. Let $B$ be an arbitrary nonempty set in a topological vector space $E$, and let $G: B \rightarrow 2^{E}$ be a KKM map. If $G(x)$ is 
closed for all $x \in B$ and is compact for at least one $x \in B$, then $\bigcap_{x \in B} G(x) \neq \varnothing$.

\section{Iterative Schemes}

We introduce the following basic algorithm framework for (2). Let $\rho$ be a positive parameter and, for a given iterate $x_{n}$, consider the auxiliary problem that consists of finding $x_{n+1}$ such that

$$
\begin{array}{r}
\left\langle\rho T\left(x_{n}\right)+h^{\prime}\left(x_{n+1}\right)-h^{\prime}\left(x_{n}\right), \eta\left(y, x_{n+1}\right)\right\rangle+\rho\left[f(y)-f\left(x_{n+1}\right)\right] \geq 0, \\
\text { for all } y \in K,
\end{array}
$$

where $h^{\prime}(x)$ is the Fréchet derivative of a functional $h: K \rightarrow \mathbb{R}$ at $x$.

Theorem 3.1. Let $K$ be a nonempty convex and bounded subset of a real Hilbert space $H$, and let $f: K \rightarrow \mathbb{R}$ be a lower semicontinuous and convex functional. Let $T: K \rightarrow H$ be $\eta$-cocoercive with constant $\alpha$. Assume that:

(i) $\eta: K \times K \rightarrow H$ is Lipschitz continuous with constant $\lambda$ such that

(a) $\eta(x, y)+\eta(y, x)=0$, for all $x, y \in K$,

(b) $\eta(x, y)=\eta(x, z)+\eta(z, y)$, for all $x, y, z \in K$,

(c) $\eta(\cdot, \cdot)$ is affine in the first variable,

(d) for each fixed $y \in K, x \mapsto \eta(y, x)$ is sequentially continuous from the weak topology to the weak topology;

(ii) $\quad h: K \rightarrow \mathbb{R}$ is $\eta$-strongly convex with constant $\mu$ and its derivative $h^{\prime}$ is sequentially continuous from the weak topology to the strong topology;

(iii) there exists $\gamma>0$ such that, for any $x, y \in K$,

$$
h(y)-h(x)-\left\langle h^{\prime}(x), \eta(y, x)\right\rangle \leq \gamma\|y-x\|^{2} .
$$

Then, there exists a unique solution $x_{n+1} \in K$ to (3). If

$$
0<\rho<2 \alpha \mu / \lambda^{2},
$$

then the sequence $\left\{x_{n}\right\}$ generated by (3) converges to a solution of (2).

Proof. Existence of Solutions of Problem (3). For the sake of simplicity, we write (3) as follows: Find $\bar{x} \in K$ such that

$\left\langle\rho T\left(x_{n}\right)+h^{\prime}(\bar{x})-h^{\prime}\left(x_{n}\right), \eta(y, \bar{x})\right\rangle+\rho[f(y)-f(\bar{x})] \geq 0, \quad$ for all $y \in K$. 
For each fixed $n$ and each $y \in K$, we define $G(y)=\left\{x \in K:\left\langle\rho T\left(x_{n}\right)+h^{\prime}(x)-h^{\prime}\left(x_{n}\right), \eta(y, x)\right\rangle+\rho[f(y)-f(x)] \geq 0\right\}$.

Note that, for each $y \in K, G(y)$ is nonempty, since $y \in G(y)$.

We shall prove that $G$ is a KKM map. Suppose that there is a finite subset $\left\{y_{1}, y_{2}, \ldots, y_{k}\right\}$ of $K$ and that $\alpha_{i} \geq 0$, for all $i=1,2, \ldots, k$ with $\sum_{i=1}^{k} \alpha_{i}=1$ such that

$$
\hat{x}=\sum_{i=1}^{k} \alpha_{i} y_{i} \notin G\left(y_{i}\right), \quad \text { for all } i .
$$

Then, we have

$$
\left\langle\rho T\left(x_{n}\right)+h^{\prime}(\hat{x})-h^{\prime}\left(x_{n}\right), \eta\left(y_{i}, \hat{x}\right)\right\rangle+\rho\left[f\left(y_{i}\right)-f(\hat{x})\right]<0, \quad \text { for all } i .
$$

Therefore,

$$
\sum_{i=1}^{k} \alpha_{i}\left\langle\rho T\left(x_{n}\right)+h^{\prime}(\hat{x})-h^{\prime}\left(x_{n}\right), \eta\left(y_{i}, \hat{x}\right)\right\rangle+\rho \sum_{i=1}^{k} \alpha_{i}\left[f\left(y_{i}\right)-f(\hat{x})\right]<0 .
$$

From condition (i)(a), we have

$$
\eta(x, x)=0, \quad \text { for all } x \in K .
$$

By using the convexity of $f$ and assumption (i)(c), we get

$$
0=\left\langle\rho T\left(x_{n}\right)+h^{\prime}(\hat{x})-h^{\prime}\left(x_{n}\right), \eta(\hat{x}, \hat{x})\right\rangle<0,
$$

a contradiction. Hence, $G$ is a KKM map.

Since $\overline{G(y)}{ }^{w}$ [the weak closure of $G(y)$ ] is a weakly closed subset of a bounded set $K \subseteq H$, it is weakly compact. Hence, by Lemma 2.2,

$$
\bigcap_{y \in K} \overline{G(y)^{w}} \neq \varnothing .
$$

Let

$$
\bar{x} \in \bigcap_{y \in K} \overline{G(y)}^{w} .
$$

Then, there exists a sequence $\left\{x_{m}\right\}$ in $G(y)$ such that $x_{m} \rightarrow \bar{x}$; see Ref. 21, pp. 93. Then,

$$
\left\langle\rho T\left(x_{n}\right)+h^{\prime}\left(x_{m}\right)-h^{\prime}\left(x_{n}\right), \eta\left(y, x_{m}\right)\right\rangle \geq \rho\left[f\left(x_{m}\right)-f(y)\right],
$$

and hence,

$$
\lim _{m \rightarrow \infty}\left\langle\rho T\left(x_{n}\right)+h^{\prime}\left(x_{m}\right)-h^{\prime}\left(x_{n}\right), \eta\left(y, x_{m}\right)\right\rangle \geq \rho \lim _{m \rightarrow \infty}\left[f\left(x_{m}\right)-f(y)\right] .
$$


Since $f$ is convex and lower semicontinuous, it is lower semicontinuous in the weak topology. Using Lemma 2.1, we get

$$
\left\langle\rho T\left(x_{n}\right)+h^{\prime}(\bar{x})-h^{\prime}\left(x_{n}\right), \eta(y, \bar{x})\right\rangle \geq \rho[f(\bar{x})-f(y)] .
$$

Therefore, $\bar{x} \in K$ is a solution of (3).

Uniqueness of Solution of Problem (3). Let $x_{1}$ and $x_{2}$ be two solutions of (3). Then, for all $y \in K$,

$$
\begin{aligned}
& \left\langle\rho T\left(x_{n}\right)+h^{\prime}\left(x_{1}\right)-h^{\prime}\left(x_{n}\right), \eta\left(y, x_{1}\right)\right\rangle+\rho\left[f(y)-f\left(x_{1}\right)\right] \geq 0, \\
& \left\langle\rho T\left(x_{n}\right)+h^{\prime}\left(x_{2}\right)-h^{\prime}\left(x_{n}\right), \eta\left(y, x_{2}\right)\right\rangle+\rho\left[f(y)-f\left(x_{2}\right)\right] \geq 0 .
\end{aligned}
$$

Taking $y=x_{2}$ in (5) and $y=x_{1}$ in (6), and adding these inequalities, we get

$$
\begin{aligned}
& \rho\left\langle T\left(x_{n}\right), \eta\left(x_{2}, x_{1}\right)\right\rangle+\left\langle h^{\prime}\left(x_{1}\right)-h^{\prime}\left(x_{n}\right), \eta\left(x_{2}, x_{1}\right)\right\rangle \\
& +\rho\left\langle T\left(x_{n}\right), \eta\left(x_{1}, x_{2}\right)\right\rangle+\left\langle h^{\prime}\left(x_{2}\right)-h^{\prime}\left(x_{n}\right), \eta\left(x_{1}, x_{2}\right) \geq 0 .\right.
\end{aligned}
$$

Since

$$
\eta(x, y)+\eta(y, x)=0, \quad \text { for all } x, y \in K,
$$

we have

$$
\left\langle h^{\prime}\left(x_{1}\right), \eta\left(x_{2}, x_{1}\right)\right\rangle \geq-\left\langle h^{\prime}\left(x_{2}\right), \eta\left(x_{1}, x_{2}\right)\right\rangle .
$$

By using the $\eta$-strong convexity of $h$, we obtain

$$
h\left(x_{2}\right)-h\left(x_{1}\right)-(\mu / 2)\left\|x_{1}-x_{2}\right\|^{2} \geq-h\left(x_{1}\right)+h\left(x_{2}\right)+(\mu / 2)\left\|x_{2}-x_{1}\right\|^{2},
$$

and therefore,

$$
\mu\left\|x_{1}-x_{2}\right\|^{2} \leq 0 .
$$

Since $\mu>0$, we get $x_{1}=x_{2}$. Hence, the solution of (3) is unique.

Let $x^{*}$ be any fixed solution of (2). For each $y \in K$, we define a functional

$$
\Lambda(y)=h\left(x^{*}\right)-h(y)-\left\langle h^{\prime}(y), \eta\left(x^{*}, y\right)\right\rangle .
$$

By the $\eta$-strong convexity of $h$, we have

$$
\Lambda(y)=h\left(x^{*}\right)-h(y)-\left\langle h^{\prime}(y), \eta\left(x^{*}, y\right)\right\rangle \geq(\mu / 2)\left\|y-x^{*}\right\|^{2} .
$$

From the $\eta$-strong convexity of $h$, assumption (i)(b), and (3) with $y=x^{*}$, we get

$$
\begin{aligned}
& \Lambda\left(x_{n}\right)-\Lambda\left(x_{n+1}\right) \\
& =h\left(x_{n+1}\right)-h\left(x_{n}\right)-\left\langle h^{\prime}\left(x_{n}\right), \eta\left(x^{*}, x_{n}\right)\right\rangle+\left\langle h^{\prime}\left(x_{n+1}\right), \eta\left(x^{*}, x_{n+1}\right)\right\rangle
\end{aligned}
$$




$$
\begin{aligned}
= & h\left(x_{n+1}\right)-h\left(x_{n}\right)-\left\langle h^{\prime}\left(x_{n}\right), \eta\left(x^{*}, x_{n+1}\right)\right\rangle \\
& -\left\langle h^{\prime}\left(x_{n}\right), \eta\left(x_{n+1}, x_{n}\right)\right\rangle+\left\langle h^{\prime}\left(x_{n+1}\right), \eta\left(x^{*}, x c_{n+1}\right)\right\rangle \\
= & h\left(x_{n+1}\right)-h\left(x_{n}\right) \\
& -\left\langle h^{\prime}\left(x_{n}\right), \eta\left(x_{n+1}, x_{n}\right)\right\rangle+\left\langle h^{\prime}\left(x_{n+1}\right)-h^{\prime}\left(x_{n}\right), \eta\left(x^{*}, x_{n+1}\right)\right\rangle \\
\geq & (\mu / 2)\left\|x_{n}-x_{n+1}\right\|^{2}+\left\langle h^{\prime}\left(x_{n+1}\right)-h^{\prime}\left(x_{n}\right), \eta\left(x^{*}, x_{n+1}\right)\right\rangle \\
\geq & (\mu / 2)\left\|x_{n}-x_{n+1}\right\|^{2}-\rho\left\langle T\left(x_{n}\right), \eta\left(x^{*}, x_{n+1}\right)\right\rangle-\rho\left[f\left(x^{*}\right)-f\left(x_{n+1}\right)\right] \\
= & (\mu / 2)\left\|x_{n}-x_{n+1}\right\|^{2} \\
& +\rho\left\langle T\left(x_{n}\right), \eta\left(x_{n+1}, x^{*}\right)\right\rangle+\rho\left[f\left(x_{n+1}\right)-f\left(x^{*}\right)\right] .
\end{aligned}
$$

We set $y=x_{n+1}$ in (2) and combine it with (8); we get

$$
\begin{aligned}
\Lambda\left(x_{n}\right)-\Lambda\left(x_{n+1}\right) \geq & (\mu / 2)\left\|x_{n}-x_{n+1}\right\|^{2} \\
& +\rho\left\langle T\left(x_{n}\right), \eta\left(x_{n+1}, x^{*}\right)\right\rangle-\rho\left\langle T\left(x^{*}\right), \eta\left(x_{n+1}, x^{*}\right)\right\rangle \\
= & (\mu / 2)\left\|x_{n}-x_{n+1}\right\|^{2}+Q .
\end{aligned}
$$

Now,

$$
\begin{aligned}
Q & =\rho\left\langle T\left(x_{n}\right)-T\left(x^{*}\right), \eta\left(x_{n+1}, x^{*}\right)\right\rangle \\
& =\rho\left\langle T\left(x_{n}\right)-T\left(x^{*}\right), \eta\left(x_{n}, x^{*}\right)\right\rangle+\rho\left\langle T\left(x_{n}\right)-T\left(x^{*}\right), \eta\left(x_{n+1}, x_{n}\right)\right\rangle \\
& \geq \rho\left[\alpha\left\|T\left(x_{n}\right)-T\left(x^{*}\right)\right\|^{2}+\left\langle T\left(x_{n}\right)-T\left(x^{*}\right), \eta\left(x_{n+1}, x_{n}\right)\right\rangle\right] \\
& \geq \rho\left[-(1 / 4 \alpha)\left\|\eta\left(x_{n+1}, x_{n}\right)\right\|^{2}\right] \\
& \geq\left(-\rho \lambda^{2} / 4 \alpha\right)\left\|x_{n+1}-x_{n}\right\|^{2} .
\end{aligned}
$$

Therefore,

$$
\Lambda\left(x_{n}\right)-\Lambda\left(x_{n+1}\right) \geq(1 / 2)\left(\mu-\rho \lambda^{2} / 2 \alpha\right)\left\|x_{n+1}-x_{n}\right\|^{2} .
$$

If $x_{n+1}=x_{n}$ for some $n$, then $x_{n}$ is a solution of (2). Otherwise, it follows from (4) that $\Lambda\left(x_{n}\right)-\Lambda\left(x_{n+1}\right)$ is a nonnegative sequence, from which we have

$$
\lim _{n \rightarrow \infty}\left\|x_{n+1}-x_{n}\right\|=0
$$

Also from (7), we conclude that $\left\{\Lambda\left(x_{n}\right)\right\}$ is a decreasing sequence, and hence $\left\{x_{n}\right\}$ must be a bounded sequence. It is easy to see that any cluster point of $\left\{x_{n}\right\}$ is a solution of (2).

Now, let $\bar{x}$ be any cluster point of $\left\{x_{n}\right\}$, and let

$$
\bar{\Lambda}\left(x_{n}\right)=h(\bar{x})-h\left(x_{n}\right)-\left\langle h^{\prime}\left(x_{n}\right), \eta\left(\bar{x}, x_{n}\right)\right\rangle \geq(\mu / 2)\left\|\bar{x}-x_{n}\right\|^{2} .
$$


By the above argument, we know that $\left\{\bar{\Lambda}\left(x_{n}\right)\right\}$ is also a decreasing sequence; by assumption (iii), we have

$$
\bar{\Lambda}\left(x_{n}\right) \leq \gamma\left\|\bar{x}-x_{n}\right\|^{2},
$$

from which it follows that

$$
\lim _{n \rightarrow \infty} \bar{\Lambda}\left(x_{n}\right)=0 .
$$

Combining (10) and (11), we conclude that the sequence $\left\{x_{n}\right\}$ converges to $\bar{x}$.

Remark 3.1. When $\eta(y, x)=y-x$ for all $x, y \in K$, Theorem 3.1 is an infinite-dimensional version of Theorem 3.2 in Ref. 15.

We now have the following basic algorithm framework for the (MVLIP) (1).

Let $\rho$ be a positive parameter and, for a given iterate $x_{n}$, consider the auxiliary (MVLIP) that consists of finding $x_{n+1}$ such that

$$
\begin{aligned}
& \left\langle\rho\left(T\left(x_{n}\right)-A\left(x_{n}\right)\right)+h^{\prime}\left(x_{n+1}\right)-h^{\prime}\left(x_{n}\right), \eta\left(y, x_{n+1}\right)\right\rangle \\
& +\rho\left[f(y)-f\left(x_{n+1}\right)\right] \geq 0, \quad \text { for all } y \in K,
\end{aligned}
$$

where $h^{\prime}(x)$ is the Fréchet derivative of a functional $h: K \rightarrow \mathbb{R}$ at $x$.

Theorem 3.2. Let $K$ be a nonempty convex and bounded subset of a real Hilbert space $H$, and let $f: K \rightarrow \mathbb{R}$ be a lower semicontinuous and convex functional. Let $T, A: K \rightarrow H$ be two maps such that $T$ is $\eta$-cocoercive with constant $\alpha$ and $A$ is $\eta$-relaxed monotone with constant $\xi$ and Lipschitz continuous with constant $\beta$. Assume that:

(i) $\eta: K \times K \rightarrow H$ is Lipschitz continuous with constant $\lambda$ such that

(a) $\eta(x, y)+\eta(y, x)=0$, for all $x, y \in K$,

(b) $\eta(x, y)=\eta(x, z)+\eta(z, y)$, for all $x, y, z \in K$,

(c) $\eta(\cdot, \cdot)$ is affine in the first variable,

(d) for each fixed $y \in K, x \mapsto \eta(y, x)$ is sequentially continuous from the weak topology to the weak topology;

(ii) $h: K \rightarrow \mathbb{R}$ is $\eta$-strongly convex with constant $\mu$, and its derivative $h^{\prime}$ is sequentially continuous from the weak topology to the strong topology.

Then, there exists a unique solution $x_{n+1} \in K$ to (12). If

$$
0<\rho<2 \alpha \mu \xi / \lambda^{2}\left(\xi+\alpha \beta^{2}\right),
$$


then the sequence $\left\{x_{n}\right\}$ generated by (12) converges to a solution of the (MVLIP)(1).

Proof. The first part follows by replacing $T$ with $T-A$ in the proof of the first part of Theorem 3.1.

Let $x^{*}$ be any fixed solution of (1). For each $y \in K$, we define a functional

$$
\Lambda(y)=h\left(x^{*}\right)-h(y)-\left\langle h^{\prime}(y), \eta\left(x^{*}, y\right)\right\rangle .
$$

From the $\eta$-strong convexity of $h$, assumption (i)(b), and (12) with $y=x^{*}$, we get

$$
\begin{aligned}
\Lambda & \left(x_{n}\right)-\Lambda\left(x_{n+1}\right) \\
= & h\left(x_{n+1}\right)-h\left(x_{n}\right)-\left\langle h^{\prime}\left(x_{n}\right), \eta\left(x^{*}, x_{n}\right)\right\rangle+\left\langle h^{\prime}\left(x_{n+1}\right), \eta\left(x^{*}, x_{n+1}\right)\right\rangle \\
= & h\left(x_{n+1}\right)-h\left(x_{n}\right)-\left\langle h^{\prime}\left(x_{n}\right), \eta\left(x^{*}, x_{n+1}\right)\right\rangle \\
- & \left\langle h^{\prime}\left(x_{n}\right), \eta\left(x_{n+1}, x_{n}\right)\right\rangle+\left\langle h^{\prime}\left(x_{n+1}\right), \eta\left(x^{*}, x_{n+1}\right)\right\rangle \\
= & h\left(x_{n+1}\right)-h\left(x_{n}\right)-\left\langle h^{\prime}\left(x_{n}\right), \eta\left(x_{n+1}, x_{n}\right)\right\rangle \\
& +\left\langle h^{\prime}\left(x_{n+1}\right)-h^{\prime}\left(x_{n}\right), \eta\left(x^{*}, x_{n+1}\right)\right\rangle \\
\geq & (\mu / 2)\left\|x_{n}-x_{n+1}\right\|^{2}+\left\langle h^{\prime}\left(x_{n+1}\right)-h^{\prime}\left(x_{n}\right), \eta\left(x^{*}, x_{n+1}\right)\right\rangle \\
\geq & (\mu / 2)\left\|x_{n}-x_{n+1}\right\|^{2} \\
& -\rho\left\langle T\left(x_{n}\right)-A\left(x_{n}\right), \eta\left(x^{*}, x_{n+1}\right)\right\rangle-\rho\left[f\left(x^{*}\right)-f\left(x_{n+1}\right)\right] \\
= & (\mu / 2)\left\|x_{n}-x_{n+1}\right\|^{2} \\
& +\rho\left\langle T\left(x_{n}\right)-A\left(x_{n}\right), \eta\left(x_{n+1}, x^{*}\right)\right\rangle+\rho\left[f\left(x_{n+1}\right)-f\left(x^{*}\right)\right] .
\end{aligned}
$$

We set $y=x_{n+1}$ in (1) and combine it with (14); we get

$$
\begin{aligned}
& \Lambda\left(x_{n}\right)-\Lambda\left(x_{n+1}\right) \\
& \geq(\mu / 2)\left\|x_{n}-x_{n+1}\right\|^{2}+\rho\left\langle T\left(x_{n}\right)-A\left(x_{n}\right), \eta\left(x_{n+1}, x^{*}\right)\right\rangle \\
& \quad-\rho\left\langle T\left(x^{*}\right)-A\left(x^{*}\right), \eta\left(x_{n+1}, x^{*}\right)\right\rangle \\
& =(\mu / 2)\left\|x_{n}-x_{n+1}\right\|^{2}+Q .
\end{aligned}
$$

Now,

$$
\begin{aligned}
Q= & \rho\left\langle T\left(x_{n}\right)-T\left(x^{*}\right), \eta\left(x_{n+1}, x^{*}\right)\right\rangle-\rho\left\langle A\left(x_{n}\right)-A\left(x^{*}\right), \eta\left(x_{n+1}, x^{*}\right)\right\rangle \\
= & \rho\left\langle T\left(x_{n}\right)-T\left(x^{*}\right), \eta\left(x_{n}, x^{*}\right)\right\rangle+\rho\left\langle T\left(x_{n}\right)-T\left(x^{*}\right), \eta\left(x_{n+1}, x_{n}\right)\right\rangle \\
& -\rho\left\langle A\left(x_{n}\right)-A\left(x^{*}\right), \eta\left(x_{n}, x^{*}\right)\right\rangle-\rho\left\langle A\left(x_{n}\right)-A\left(x^{*}\right), \eta\left(x_{n+1}, x_{n}\right)\right\rangle
\end{aligned}
$$


Therefore,

$$
\begin{aligned}
\geq & \rho \alpha\left\|T\left(x_{n}\right)-T\left(x^{*}\right)\right\|^{2}+\rho\left\langle T\left(x_{n}\right)-T\left(x^{*}\right), \eta\left(x_{n+1}, x_{n}\right)\right\rangle \\
& +\rho \xi\left\|x_{n}-x^{*}\right\|^{2}-\rho\left\|A\left(x_{n}\right)-A\left(x^{*}\right)\right\| \cdot\left\|\eta\left(x_{n+1}, x_{n}\right)\right\| \\
\geq & \rho\left[\alpha\left\|T\left(x_{n}\right)-T\left(x^{*}\right)\right\|^{2}+\left\langle T\left(x_{n}\right)-T\left(x^{*}\right), \eta\left(x_{n+1}, x_{n}\right)\right\rangle\right] \\
& +\rho \xi\left\|x_{n}-x^{*}\right\|^{2}-\rho \beta \lambda\left\|x_{n}-x^{*}\right\| \cdot\left\|x_{n+1}-x_{n}\right\| \\
\geq & \rho\left[-(1 / 4 \alpha)\left\|\eta\left(x_{n+1}, x_{n}\right)\right\|^{2}\right] \\
& +\rho \xi\left\|x_{n}-x^{*}\right\|^{2}-\rho \beta \lambda\left\|x_{n}-x^{*}\right\| \cdot\left\|x_{n+1}-x_{n}\right\| \\
\geq & \left(-\rho \lambda^{2} / 4 \alpha\right)\left\|x_{n+1}-x_{n}\right\|^{2} \\
& +\rho \xi\left\|x_{n}-x^{*}\right\|^{2}-\rho \beta \lambda\left\|x_{n}-x^{*}\right\| \cdot\left\|x_{n+1}-x_{n}\right\|
\end{aligned}
$$

$$
\begin{aligned}
& \Lambda\left(x_{n}\right)-\Lambda\left(x_{n+1}\right) \\
& \geq(1 / 2)\left(\mu-\rho \lambda^{2} / 2 \alpha\right)\left\|x_{n+1}-x_{n}\right\|^{2} \\
& +\rho \xi\left\|x_{n}-x^{*}\right\|^{2}-\rho \beta \lambda\left\|x_{n}-x^{*}\right\| \cdot\left\|x_{n+1}-x_{n}\right\| \\
& \geq-\left[\rho^{2} \beta^{2} \lambda^{2} / 2\left(\mu-\rho \lambda^{2} / 2 \alpha\right)\right]\left\|x_{n}-x^{*}\right\|^{2}+\rho \xi\left\|x_{n}-x^{*}\right\|^{2} \\
& \geq\left[\rho \xi-\rho^{2} \beta^{2} \lambda^{2} / 2\left(\mu-\rho \lambda^{2} / 2 \alpha\right)\right]\left\|x_{n}-x^{*}\right\|^{2} .
\end{aligned}
$$

Condition (13) and inequality (15) show that the sequence $\left\{\Lambda\left(x_{n}\right)\right\}$ is strictly decreasing (unless $x_{n}=x^{*}$ ) and is nonnegative by (7). Hence, it converges to some number. Therefore, the difference of two successive terms of the sequence goes to zero, and so the sequence $\left\{x_{n}\right\}$ converges strongly to $x^{*}$ as $n \rightarrow \infty$.

Remark 3.2. The boundedness assumption on $K$ can be replaced with the following condition:

there exist a bounded subset $C$ of $K$ and $y_{0} \in C$ such that, for any $x \in K \backslash C$,

$$
\left\langle\rho\left(T\left(x_{n}\right)-A\left(x_{n}\right)\right)+h^{\prime}(x)-h^{\prime}\left(x_{n}\right), \eta\left(y_{0}, x\right)\right\rangle+\rho\left(f\left(y_{0}\right)-f(x)\right)<0 .
$$

For further details, we refer to Ref. 22.

As Zhu and Marcotte noticed in Ref. 15, from a practical point of view, the solution of the auxiliary (MVLIP) cannot be obtained in closed form and a trade-off must be achieved between the amount of work spent on solving the auxiliary problem and the accuracy of the corresponding solution. More precisely, at step $n$, one can find an approximate solution of 
the auxiliary problem, that is, a point $x_{n+1} \in K$ such that

$$
\begin{aligned}
& \left\langle\rho\left(T\left(x_{n}\right)-A\left(x_{n}\right)\right)+h^{\prime}\left(x_{n+1}\right)-h^{\prime}\left(x_{n}\right), \eta\left(y, x_{n+1}\right)\right\rangle \\
& +\rho\left[f(y)-f\left(x_{n+1}\right)\right] \geq-\epsilon_{n}, \quad \text { for all } y \in K^{\prime},
\end{aligned}
$$

where $\epsilon_{n}>0$. If $K$ is bounded, we let $K^{\prime}=K$. Otherwise, we define

$$
K^{\prime}=K \cap\{x:\|x\| \leq R\},
$$

where $R$ is a suitably large constant. We note that such a number always exists because $\left\{\left\|x_{n}-x^{*}\right\|\right\}$ is bounded.

Theorem 3.3. Assume that all the conditions of Theorem 3.2 are satisfied, and let $\left\{\boldsymbol{\epsilon}_{n}\right\}$ be a sequence such that

$$
\epsilon_{n} \geq 0 \text { and } \lim _{n \rightarrow \infty} \epsilon_{n}=0 .
$$

Then, the sequence $\left\{x_{n}\right\}$ generated by (16) converges to a solution of the (MVLIP) (1).

Proof. From the proof of Theorem 3.2, we have

$$
\begin{aligned}
& \Lambda\left(x_{n}\right)-\Lambda\left(x_{n+1}\right) \geq \theta\left\|x_{n}-x^{*}\right\|^{2}+\epsilon_{n}, \\
& \text { where } \theta=\left[\rho \xi-\rho^{2} \beta^{2} \lambda^{2} / 2\left(\mu-\rho \lambda^{2} / 2 \alpha\right)\right],
\end{aligned}
$$

and

$$
\lim _{n \rightarrow \infty}\left(\theta\left\|x_{n}-x^{*}\right\|^{2}+\epsilon_{n}\right)=0 .
$$

Now,

$$
\begin{aligned}
\lim _{n \rightarrow \infty} \theta\left\|x_{n}-x^{*}\right\|^{2} & =\lim _{n \rightarrow \infty}\left[\left(\theta\left\|x_{n}-x^{*}\right\|^{2}+\epsilon_{n}\right)-\epsilon_{n}\right] \\
& =\lim _{n \rightarrow \infty}\left(\theta\left\|x_{n}-x^{*}\right\|^{2}+\epsilon_{n}\right)-\lim _{n \rightarrow \infty} \epsilon_{n} \\
& =0,
\end{aligned}
$$

and hence $\left\{x_{n}\right\}$ converges strongly to $x^{*}$, a solution of the (MVLIP) (1).

When

$$
\eta(x, y)=x-y, \quad \text { for all } x, y \in K,
$$

assumption (iii) in Theorem 3.1 holds if $h^{\prime}$ is Lipschitz continuous on $K$. In this case, we have the following algorithm framework.

Let $\rho$ be a positive parameter and, for a given iterate $x_{n}$, consider the auxiliary mixed variational inequality problem (in short, MVIP) that 
consists of finding $x_{n+1}$ such that

$$
\begin{aligned}
& \left\langle\rho\left(T\left(x_{n}\right)-A\left(x_{n}\right)\right)+h^{\prime}\left(x_{n+1}\right)-h^{\prime}\left(x_{n}\right), y-x_{n+1}\right\rangle \\
& +\rho\left(f(y)-f\left(x_{n+1}\right)\right) \geq 0, \quad \text { for all } y \in K,
\end{aligned}
$$

where $h^{\prime}(x)$ is the Fréchet derivative of a functional $h: K \rightarrow \mathbb{R}$ at $x$.

Corollary 3.1. Let $K$ be a nonempty convex and bounded subset of a real Hilbert space $H$, and let $f: K \rightarrow \mathbb{R}$ be a lower semicontinuous and convex functional. Let $T, A: K \rightarrow H$ be two maps such that $T$ is cocoercive with constant $\alpha$ and $A$ is relaxed monotone with constant $\xi$ and Lipschitz continuous with constant $\beta$. Assume that $h: K \rightarrow \mathbb{R}$ is strongly convex with constant $\mu$ such that its derivative $h^{\prime}$ is sequentially continuous from the weak topology to the strong topology. Then, there exists a unique solution $x_{n+1} \in K$ to (17). If

$$
0<\rho<2 \alpha \mu \xi /\left(\xi+\alpha \beta^{2}\right),
$$

then the sequence $\left\{x_{n}\right\}$ generated by (17) converges to a solution of the following mixed variational inequality problem (MVIP): Find $x^{*} \in K$ such that

$$
\left\langle T\left(x^{*}\right)-A\left(x^{*}\right), y-x^{*}\right\rangle+f(y)-f\left(x^{*}\right) \geq 0, \quad \text { for all } y \in K \text {. }
$$

Corollary 3.2. Let $K$ be a nonempty convex and bounded subset of $\mathbb{R}^{n}$, and let $f: K \rightarrow \mathbb{R}$ be a lower semicontinuous and convex functional. Let $T, A: K \rightarrow \mathbb{R}^{n}$ be two maps such that $T$ is $\eta$-cocoercive with constant $\alpha$ and $A$ is $\eta$-relaxed monotone with constant $\xi$ and Lipschitz continuous with constant $\beta$. Assume that:

(i) $\eta: K \times K \rightarrow \mathbb{R}^{n}$ is Lipschitz continuous with constant $\lambda$ such that

(a) $\eta(x, y)+\eta(y, x)=0$, for all $x, y \in K$,

(b) $\eta(x, y)=\eta(x, z)+\eta(z, y)$, for all $x, y, z \in K$,

(c) $\eta(\cdot, \cdot)$ is affine in the first variable,

(d) for each $y \in K, x \mapsto \eta(y, x)$ is continuous on $K$;

(ii) $h: K \rightarrow \mathbb{R}$ is continuously differentiable and $\eta$-strongly convex with constant $\mu$.

Then, there exists a unique solution $x_{n+1} \in K$ to (12). If (13) holds, then the sequence $\left\{x_{n}\right\}$ generated by (12) converges to a solution of the (MVLIP) (1).

Corollary 3.3. Let $K$ be a nonempty convex and bounded subset of $\mathbb{R}^{n}$, and let $f: K \rightarrow \mathbb{R}$ be a lower semicontinuous and convex functional. Let 
$T, A: K \rightarrow \mathbb{R}^{n}$ be two maps such that $T$ is cocoercive with constant $\alpha$ and $A$ is $\eta$-relaxed monotone with constant $\xi$ and Lipschitz continuous with constant $\beta$. Assume that $h: K \rightarrow \mathbb{R}$ is continuously differentiable and strongly convex with constant $\mu$. Then, there exists a unique solution $x_{n+1} \in K$ to (17). If (18) holds, then the sequence $\left\{x_{n}\right\}$ generated by (17) converges to a solution of the (MVLIP) (19).

\section{References}

1. Ding, X. P., Random Mixed Variational-Like Inequalities in Topological Vector Spaces, Journal of the Sichuan Normal University, Vol. 20, pp. 1-13, 1997.

2. Noor, M. A., Nonconvex Functions and Variational Inequalities, Journal of Optimization Theory and Applications, Vol. 87, pp. 615-630, 1995.

3. Dien, N. H., Some Remarks on Variational-Like and Quasi-Variational-Like Inequalities, Bulletin of the Australian Mathematical Society, Vol. 46, pp. 335342, 1992.

4. Noor, M. A., Variational-Like Inequalities, Optimization, Vol. 30, pp. 323-330, 1994.

5. Ansari, Q. H., and Yao, J. C., Prevariational Inequalities in Banach Spaces, Optimization: Techniques and Applications, Edited by L. Cacetta et al., Curtin University of Technology, Perth, Australia, Vol. 2, pp. 1165-1172, 1998.

6. Ansari, Q. H., and Yao, J. C., Nonlinear Variational Inequalities for Pseudomonotone Operators with Applications, Advances in Nonlinear Variational Inequalities, Vol. 3, pp. 61-69, 2000.

7. Parida, J., Sahoo, M., and Kumar, A., A Variational-Like Inequality Problem, Bulletin of the Australian Mathematical Society, Vol. 39, pp. 225-231, 1989.

8. Siddiqi, A. H., Khaliq, A., and Ansari, Q. H., On Variational-Like Inequalities, Annales des Sciences Mathématiques du Québec, Vol. 18, pp. 39-48, 1994.

9. Yang, X. Q., and Chen, G. Y., A Class of Nonconvex Functions and Prevariational Inequalities, Journal of Mathematical Analysis and Applications, Vol. 169, pp. 359-373, 1992.

10. Cohen, G., Optimization by Decomposition and Coordination: A Unified Approach, IEEE Transactions on Automatic Control, Vol. 23, pp. 222-232, 1978.

11. Cohen, G., Auxiliary Problem Principle and Decomposition of Optimization Problems, Journal of Optimization Theory and Applications, Vol. 32, pp. 277305, 1980.

12. Cohen, G., and Zhu, D. L., Decomposition Coordination Methods in LargeScale Optimization Problems: The Nondifferentiable Case and the Use of Augmented Lagrangians, Advances in Large-Scale Systems: Theory and Applications, Edited by J. B. Cruz, JAI Press, Greenwich, Connecticut, Vol. 1, pp. 203-266, 1984. 
13. Cohen, G., Auxiliary Problem Principle Extended to Variational Inequalities, Journal of Optimization Theory and Applications, Vol. 49, pp. 325-333, 1988.

14. Glowinski, R., Lions, J. L., and Trémolières, R., Numerical Analysis of Variational Inequalities, North-Holland, Amsterdam, Holland, 1981.

15. Zhu, D. L., and Marcotte, P., Cocoercivity and Its Role in the Convergence of Iterative Schemes for Solving Variational Inequalities, SIAM Journal on Optimization, Vol. 6, pp. 714-726, 1996.

16. Tseng, P., Further Applications of a Splitting Algorithm to Decomposition in Variational Inequalities and Convex Programming, Mathematical Programming, Vol. 48, pp. 249-263, 1990.

17. Karamardian, S., The Nonlinear Complementarity Problem with Applications, Part 2, Journal of Optimization Theory and Applications, Vol. 4, pp. 167-181, 1969.

18. Hanson, M. A., On Sufficiency of the Kuhn-Tucker Conditions, Journal of Mathematical Analysis and Applications, Vol. 80, pp. 545-550, 1981.

19. Kӧтне, G., Topological Vector Spaces, I, Springer Verlag, Berlin, Germany, 1983.

20. FAn, K., A Generalization of Tychonoff's Fixed-Point Theorem, Mathematische Annalen, Vol. 142, pp. 305-310, 1961.

21. Deimling, K., Nonlinear Functional Analysis, Springer Verlag, Berlin, Germany, 1985.

22. Yао, J. C., Multivalued Variational Inequalities with K-Pseudomonotone Operators, Journal of Optimization Theory and Applications, Vol. 83, pp. 391403, 1994. 